Conclusion LIN led to significant absolute and \% improvement in $\mathrm{AP}$ vs $\mathrm{PBO}$ overall and stratified by baseline AP severity. Absolute magnitude of improvement in AP significantly correlated with baseline AP severity; however, all groups had similar \% improvement in AP. Pt-rated relief of AP was consistent across baseline AP severity groups. Support: Ironwood Pharmaceuticals Inc \& Forest Laboratories Inc. Editing: CMC funded by Almirall

Disclosure of Interest J. Johnston Shareholder of: Ironwood Pharmaceuticals, Employee of: Ironwood Pharmaceuticals, W. Chey Consultant for: Ironwood Pharmaceuticals and Forest Laboratories, B. Lavins Shareholder of: Ironwood Pharmaceuticals, Employee of: Ironwood Pharmaceuticals, S. Shiff Shareholder of: Forest Laboratories, Employee of: Forest Laboratories, C. Kurtz Shareholder of: Ironwood Pharmaceuticals, Employee of: Ironwood Pharmaceuticals, J. MacDougall Shareholder of: Ironwood Pharmaceuticals, Employee of: Ironwood Pharmaceuticals, X. Jia Shareholder of: Forest Laboratories, Employee of: Forest Laboratories, H. Schneier Shareholder of: Forest Laboratories, Employee of: Forest Laboratories, M. Currie Shareholder of: Ironwood Pharmaceuticals, Employee of: Ironwood Pharmaceuticals

\section{PWE-029 CHARACTERISATION AND ASSOCIATION OF ABDOMINAL PAIN WITH ANXIETY OR DEPRESSION IN PATIENTS WITH IRRITABLE BOWEL SYNDROME WITH CONSTIPATION (IBS-C)}

doi:10.1136/gutjnl-2013-304907.318

'E M Quigley, ${ }^{2 B}$ Lavins, ${ }^{3}$ S J Shiff, ${ }^{3} \mathrm{~K}$ Shi, ${ }^{2} \mathrm{X}$ Hao, ${ }^{2} \mathrm{C}$ Kurtz, ${ }^{2} \mathrm{M}$ Currie, ${ }^{3} \mathrm{H}$ Schneier, 2,"J Johnston. 'Alimentary Pharmabiotic Centre, Cork, Ireland; ${ }^{2}$ Ironwood Pharmaceuticals, Cambridge; ${ }^{3}$ Forest Research Institute, Jersey City, United States

Introduction The Short-Form McGill Pain Questionnaire (SFMPQ-2) assesses and characterises pain. It consists of 22 items (rated from $0=$ none to $10=$ worst possible) in 4 subscales (continuous pain, intermittent pain, neuropathic pain [sensory descriptors], and affective descriptors [emotional aspects of pain, eg cruel/exhausting]). The SF-MPQ-2 has not yet been validated in abdominal pain and, therefore, its use in functional gastrointestinal disorders like IBS is limited. Also, little is known about pain quality in IBS. We used the SF-MPQ-2 to characterise baseline abdominal pain in IBS-C and to determine whether subscale scores were associated with significant baseline anxiety or depression.

Methods Over a 2-wk baseline period in 2 Phase 3 trials of linaclotide (LIN), patients (pts) with IBS-C (Rome II criteria; $N=1523$ ) rated daily their worst abdominal pain over the past $24 \mathrm{~h}$ on an 11 -point scale $(0=$ none, $10=$ very severe $)$ and completed the SFMPQ-2. Summary statistics were calculated for each SF-MPQ-2 item and subscale. Pts were grouped by their highest-scored pain subscale and the pain subscale reported by the highest \% of pts was defined as the predominant pain type. Association of each subscale with baseline abdominal pain score was determined by ANCOVA. Baseline anxiety and depression were assessed on the Hospital Anxiety and Depression Scale (HADS-A and HADS-D); pts were categorised as normal/borderline (0-10) or abnormal (11-21). Association of each subscale with abnormal HADS was analysed by logistic regression.

Results Continuous pain was the predominant pain type $77 \%$ of pts); the item with the highest average score in this subscale was cramping pain. Baseline abdominal pain score was significantly associated with McGill continuous pain $(p<0.0001)$, intermittent pain $(p=0.004)$ and affective descriptors $(p=0.012)$, but not with neuropathic pain $(p=0.526)$. Only the affective descriptors subscale was significantly associated with abnormal HADS score (Table)

\section{Abstract PWE-029 Table}

\begin{tabular}{llllll}
\hline & & \multicolumn{2}{l}{ Odds ratio } & \\
\cline { 3 - 4 } Abnormal HADS & Pain subscale & Point estimate & $\mathbf{9 5 \%} \mathbf{~ C l}$ & $\mathbf{p}^{\mathbf{a}}$ \\
\hline HADS-A & Continuous & 1.04 & $0.96,1.13$ & 0.35 \\
& Intermittent & 0.96 & $0.89,1.04$ & 0.33 \\
& Neuropathic & 1.00 & $0.92,1.09$ & 0.99 \\
& Affective descriptors & 1.15 & $1.07,1.23$ & $<0.0001$ \\
HADS-D & Continuous & 1.11 & $0.94,1.30$ & 0.23 \\
& Intermittent & 0.92 & $0.80,1.05$ & 0.21 \\
& Neuropathic & 1.12 & $0.97,1.29$ & 0.12 \\
& Affective descriptors & 1.24 & $1.10,1.41$ & $<0.001$ \\
\hline
\end{tabular}

Wald $\chi^{2}$ test

Conclusion These data indicate that continuous pain is predominant in IBS-C and that anxiety and depression are related to the emotional response to pain, not to pain itself. Support: Ironwood Pharmaceuticals Inc \& Forest Laboratories Inc. Editing: CMC funded by Almirall

Disclosure of Interest E. Quigley Speaker bureau with: Danone, Janssen, Procter and Gamble, sanofi-aventis, Shire and Yakult, Conflict with: Advisory boards for Almirall, Ironwood, Janssen, Norgine, Salix and Shire/Movetis, B. Lavins Shareholder of: Ironwood Pharmaceuticals, Employee of: Ironwood Pharmaceuticals, S. Shiff Shareholder of: Forest Laboratories, Employee of: Forest Laboratories, K. Shi Employee of: Forest Laboratories, X. Hao Shareholder of: Ironwood Pharmaceuticals, Employee of: Ironwood Pharmaceuticals, C. Kurtz Shareholder of: Ironwood Pharmaceuticals, Employee of: Ironwood Pharmaceuticals, M. Currie Shareholder of: Ironwood Pharmaceuticals, Employee of: Ironwood Pharmaceuticals, $\mathrm{H}$. Schneier Shareholder of: Forest Laboratories, Employee of: Forest Laboratories, J. Johnston Shareholder of: Ironwood Pharmaceuticals, Employee of: Ironwood Pharmaceuticals

\section{PWE-030 AUDIT OF BOWEL CANCER PRESENTATION IN A COMMUNITY HOSPITAL SETTING}

doi:10.1136/gutjnl-2013-304907.319

1."J Tharakan, 'I Tiwari, 'D Beesley. 'Endoscopy Unit, St.Michael's Hospital, Braintree, UK

Introduction Most data on bowel cancer presentation are from an acute hospital setting. There have not been many reports from a community hospital.

Our audit looked into the presentation of bowel cancer from a community hospital setting.

Methods We audited the results of bowel cancer presentation from our community hospital with data collected over a two year period from June 2010 to May 2012.

We analysed the presenting symptoms, the male:female ratio, ages and location of bowel cancer.

Results 1795 colonoscopies and 2589 flexible sigmoidoscopies were performed at our hospital during this two year period.

The total number of colonic cancers found were 87 (47 found on colonoscopy and 40 on flexible sigmoidoscopy), giving an incidence of about $2 \%$.

The ages ranged from 33 years to 86years with 49 males and 38 females.

The main presenting symptoms were analysed and were as follows.

Anaemia in 16 patients Abdominal pain in 8 patients.

Per rectal (PR) bleeding in 45 patients Change in bowel habit in 25 patients.

We followed the established norm of dividing colon cancers proximal to the splenic flexure as right colonic and distal to this as left colonic. 